\title{
Pacifastin-derived Peptides Target Tumors for Use in In Vivo Imaging
}

\author{
THEO L. SOTTERO ${ }^{1,2}$, EMILY J. GIRARD ${ }^{1}$, CARRIE MYERS ${ }^{1}$, GENE HOPPING ${ }^{1}$, \\ ANDREW J. MHYRE ${ }^{1}$ and JAMES M. OLSON ${ }^{1}$ \\ ${ }^{1}$ Clinical Research Division, Fred Hutchinson Cancer Research Center, Seattle, WA, U.S.A.; \\ ${ }^{2}$ Department of Pharmacology, University of Washington, Seattle, WA, U.S.A.
}

\begin{abstract}
Background/Aim: Developments in imaging have improved cancer diagnosis, but identification of malignant cells during surgical resection remains a challenge. The aim of this study was to investigate the pacifastin family of peptides for novel activity targeting tumor cells and the delivery of either imaging or therapeutic agents. Materials and Methods: Variants of pacifastin family peptides were generated, chemically modified and tested in human tumor xenografts. Results: A tumor-homing peptide-dye conjugate (THP1) accumulated in tumors in vivo and was internalized into cells. Examination of related peptides revealed residues critical for accumulation and allowed the engineering of improved tumor-targeting variants. A THP1-drug conjugate carrying the microtubule inhibitor, MMAE, showed limited activity in vitro and no difference compared to vehicle control in vivo. Conclusion: Although there are some obstacles to developing pacifastin-derived peptides for therapeutic activity, these optimized peptides have great promise for cancer imaging.
\end{abstract}

Radiation and chemotherapy have played critical roles in cancer therapy since the 1940's but surgery remains an essential element in the treatment of most cancers with the extent of resection playing a significant role in patient outcomes (1-3). Imaging - such as MRI and PET scans - have been invaluable in visualizing the extent of tumors prior to surgery and evaluating the potential for a successful and complete resection (4). Antibody-based imaging has proven to

This article is freely accessible online.

Correspondence to: James M. Olson, Fred Hutchinson Cancer Research Center, Clinical Research Division, 1100 Fairview Ave N, Seattle, WA 98109, U.S.A. Tel: +1 2066677955, Fax: +1 2066672917, e-mail: jolson@fredhutch.org.

Key Words: LCMI-II, pacifastin, tumor-homing peptide, cystineknotted peptide, sarcoma, lymphoma, MMAE. be critical in identifying tumor cells in ex vivo tumor sections, but to date, solid tumor imaging has generally relied on small molecular probes such as gadolinium or ${ }^{18} \mathrm{~F}$-FDG $(5,6)$. The limited use of antibody-based tumor imaging results from the challenge clinicians and researchers encountered when attempting to identify cancer-specific cell surface targets. Other challenges include the heterogeneity of target expression, increased interstitial pressure limiting solid tumor penetration, and other aspects of solid tumor physiology $(7,8)$.

As an alternative to therapeutic antibodies and antibody drug conjugates (ADCs), there has been increasing interest in the use of peptides as therapeutic delivery vehicles (9). A number of groups have identified novel tumor-homing peptides that could be used as targeting agents, but none have achieved all of the requirements for clinical utility (10). Recent clinical development of imaging agents, such as tozuleristide (aka Tumor Paint/BLZ100), seek to improve detection of solid tumors in real-time, so that surgeons can determine how to maximize tumor removal while minimizing damage to critical non-cancerous tissue (e.g. healthy brain tissue) (11). This peptide-based approach shows promise in an area where there has been a surprising dearth of antibody-based therapies. An ideal tumor-homing peptide will be proteolytically stable, will achieve sufficient pharmacokinetic properties in serum, and will hit tumor cells at a higher ratio than normal tissue (12). Cystine-knotted peptides address these challenges and are of interest for tumor delivery (13). Examples include RGD-containing peptides, such as EETI 2.5F, and chlorotoxin, a variant of which is the targeting component of tozuleristide (14-16). Differences in protein expression between cancer cells lead to variable sensitivity to targeting agents (17, 18). An expanded array of tumor-homing peptides could be used as tools for basic science research in cancer biology or developed as clinical tools that will benefit patients.

With the goal of identifying peptides with novel activity targeting tumor cells, we investigated cysteine-knotted peptides for use as imaging agents. Indeed, an LCMI-II variant delivered a fluorescent molecule to solid tumors with as much as 30-fold specificity over non-cancerous tissues. The pacifastin class of peptides, which includes LCMI-II, is known 
to have protease-targeting activity (19), and we theorized that increased protease expression driving metastasis in cancerous tissues could provide a target for these peptides. Within this class, we focused on variants of LCMI-II, a high-affinity inhibitor of the serine proteases chymotrypsin and elastase, and derived from a component of the innate immune system of the migratory locust (20-23). We investigated the potential of LCMI-II variants as molecular scaffolds to target cancer cells and to deliver not only imaging agents but also a cytotoxic payload with therapeutic potential.

\section{Materials and Methods}

Materials, cell lines and reagents. RH28 (MD Anderson, Houston, TX, USA) and Ramos, A375, A204, and A673 cells (ATCC, Manassas, VA, USA) were cultured in DMEM (Gibco, Waltham, MA, USA) supplemented with $10 \%$ fetal bovine serum (FBS) at $37^{\circ} \mathrm{C}$ in $5 \% \mathrm{CO}_{2}$.

Peptide production. All L-amino acid peptides were produced using a lentivirus-based mammalian protein expression system as described elsewhere (24). Purified peptides were assessed under non-reduced and reduced conditions by RP-HPLC and SDS-PAGE gel for purity and stored lyophilized at $-20^{\circ} \mathrm{C}$. The D-amino acid form of THP1 was ordered from Bachem and synthesized according to published protocols (21). AF647 conjugation reactions were done in $50 \mathrm{mM}$ sodium bicarbonate at $\mathrm{pH}$ 7.6. Alexa Fluor 647 NHS Ester (Thermo, Waltham, MA, USA) was resuspended at $10 \mathrm{mg} / \mathrm{ml}$ in DMSO and mixed with an equimolar amount of peptide at $2 \mathrm{mg} / \mathrm{ml}$. This reaction proceeded at room temperature for $4 \mathrm{~h}$, additional peptide was added if free dye remained and/or purification occurred via RPHPLC. Concentration was determined by using AF647 absorption at $650 \mathrm{~nm}$ with the extinction coefficient $270,000 \mathrm{~cm}^{-1} \mathrm{M}^{-1}$.

Animal studies and xenografts. All studies were conducted in accordance with protocols approved by the Institutional Animal Care and Use Committee (IACUC). Xenografts were established in athymic $\mathrm{nu} / \mathrm{nu}$ mice (Harlan) by subcutaneous injection on 5 million cells suspended in $100 \mu \mathrm{l}$ of a 1:1 mixture of serum-free media and Matrigel (BD Biosciences, Franklin Lakes, NJ, USA). Tumors were then allowed to grow to $250-500 \mathrm{~mm}^{3}$ before testing for tumor accumulation.

In vivo and ex vivo optical imaging. Mice were injected by tail vein with $100 \mu \mathrm{l}$ of peptide solution with $10 \mathrm{nmol}$ of fluorescentlylabeled sample. In the competition experiment, mice were injected with $2 \mathrm{nmol}$ of fluorescently-tagged peptide and $98 \mathrm{nmol}$ of untagged peptide. Mice were euthanized at indicated time points and imaged on an IVIS (Caliper Life Sciences, Hopkinton, MA, USA) with excitation at $640 \mathrm{~nm}$ and emission measured at $680 \mathrm{~nm}$. Confocal microscopy was done using the Zeiss LSM 780 LMO microscope on sections cut from live, unfixed RH28 flank tumors $30 \mathrm{~min}$ after IV injection of with $10 \mathrm{nmol}$ THP1-AF647.

Synthesis of ${ }^{14} \mathrm{C}$ methylated peptide. Peptides were labeled with ${ }^{14} \mathrm{C}$ methyl groups via reductive amination using ${ }^{14} \mathrm{C}$ labeled formaldehyde (25). Briefly, peptides were resuspended in water and 10x PBS was added followed by $1 \%{ }^{14} \mathrm{C}$ formaldehyde $\left(1 \mathrm{mCi}{ }^{14} \mathrm{C}\right.$ formaldehyde per $6 \mathrm{mg}$ of peptide). Sodium cyanoborohydride was added, mixed, and incubated for 4-48 h. Purification was performed using StrataX columns (Phenomenex, Torrance, CA, USA). Labeled peptide was eluted with $2 \%$ formic acid in methanol. The solvent was then evaporated with a blowdown evaporator, and assessed analytically using liquid scintillation counting and HPLC equipped with an in-line scintillation detector.

Whole body autoradiography. Frozen carcasses were allowed to off gas hexane overnight at $-20^{\circ} \mathrm{C}$ and then embedded in chilled $2 \%$ carboxymethylcellulose (Sigma Aldrich, St. Louis, MO, USA) and sectioned sagittally at a thickness of $40 \mu \mathrm{m}$ on a H/I Bright 8250 Cryostat (Hacker Instruments). Radioactive control guides were drilled into each block to verify even cutting depth along the length of the section. The radioactive guide consisted of ${ }^{14} \mathrm{C}$-Glycine (American Radiolabeled Chemicals, St. Louis, MO, USA) at 0.5 $\mu \mathrm{Ci} / \mathrm{ml}$ in $0.5 \%$ BSA (Sigma Aldrich, St. Louis, MO, USA) in PBS. Sections were collected onto 4-inch wide tape (Scotch 821, ULINE) at 2-4 depths to sample approximately 30 tissues. Collected sections were freeze dried in the cryostat for 48-72 $\mathrm{h}$ then mounted on sturdy paper and covered with a single protective layer of cellophane (Reynolds Food Service Film). Mounted sections were exposed to phosphor imager plates (Raytest) along with a radioactive standard Curve (146S-PL, ARC) for 7 days, scanned on a Raytest CR-35 at "25 $\mu \mathrm{m}$ sensitive" setting.

Cell viability. Cells were seeded in a 96-well plate at 5,000 cells per well and grown overnight at $37^{\circ} \mathrm{C}$ in $5 \% \mathrm{CO}_{2}$. Cells were then treated in triplicate with $100 \mu \mathrm{l}$ of DMEM+10\%FBS containing varying concentration of MMAE or THP $1 *$-MMAE and incubated for 3 days at $37^{\circ} \mathrm{C}$ in $5 \%$. Cell viability was measured using the CellTiter Glo Assay (Promega, Madison, WI, USA) by adding $100 \mu \mathrm{l}$ of reconstituted reagent to each well and incubating with shaking for 10 min. Fluorescence was then measured using a Synergy H4 plate reader (BioTek Instruments, Winooski, VT, USA). Cell viability was expressed as a percentage relative to untreated cells. We subtracted the background by determining the fluorescence of wells containing no cells but only media and CellTiter Glo reagent. Percent viability was reported as (sample-background)/(untreated control-background) with error bars representing standard deviation.

Statistical analysis. All data were presented as the average value \pm the SD of $n$ independent measurements. Graphs were made using GraphPad Prism. Two-tailed Student's $t$-tests were used to evaluate and statistical significance was assigned for $p$-values of $<0.05$.

\section{Results}

To identify a novel peptide for the purposes of cancer targeting, we tested a small panel of cystine-knotted peptide derivatives for their ability to express at a high-level and as a single product with fully oxidized disulfide bonds. The LCMI-II derivative THP1 passed our screening criteria and we used it as a starting scaffold for a series of peptides to optimize for tumor accumulation. To enable production and selective labeling, LCMI-II was modified at the $\mathrm{N}$-terminus, and lysine residues were replaced with arginines. The Nterminal modification allows high-efficiency isolation of cleaved LCMI-II following expression as a fusion-protein. 



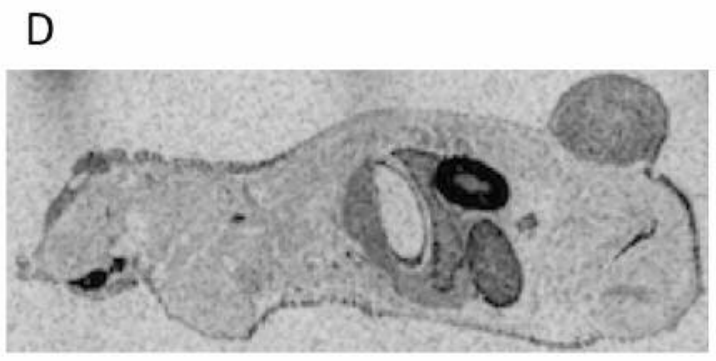

THP1* in RH28

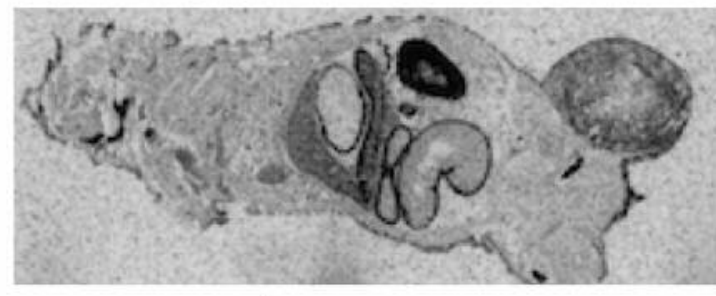

THP1*-AF647 in RH28

Figure 1. THP1 is well-folded and accumulated in human sarcoma flank xenografts. A) RP-HPLC of non-reduced (blue) and reduced (red) THP1 showing a shift in retention time upon reduction. B) SDS-PAGE of non-reduced (left) and reduced (right) THP1 demonstrating a single-product with a shift in migration upon reduction. C) Mice bearing RH28 flank xenografts were dosed with 10 nmol of AF647 or $10 \mathrm{nmol}$ of THP1-AF647 and euthanized $4 \mathrm{~h}$ following injection. Quantification of imaging signals, reported in average radiant efficiency comparing the tumor signals in the RH28 xenograft as well as the tumor:liver ratios of the mice. $n=3 . D$ ) Whole-body autoradiography images comparing the biodistribution of $T H P 1 *$ and THP1*-AF647 at $24 \mathrm{~h}$ in mice bearing RH28 flank tumors.

The lysine to arginine modifications allow selective chemical conjugation to the only remaining primary amine at the $\mathrm{N}$-terminus of the peptide. This modified form of LCMI-II, termed THP1, was expressed at a high-level as a fusion protein in mammalian cells and was easily purified following cleavage from the carrier protein. The purified THP1 peptide was a single-peak by HPLC under reducing and nonreducing conditions, suggesting a single species with a 


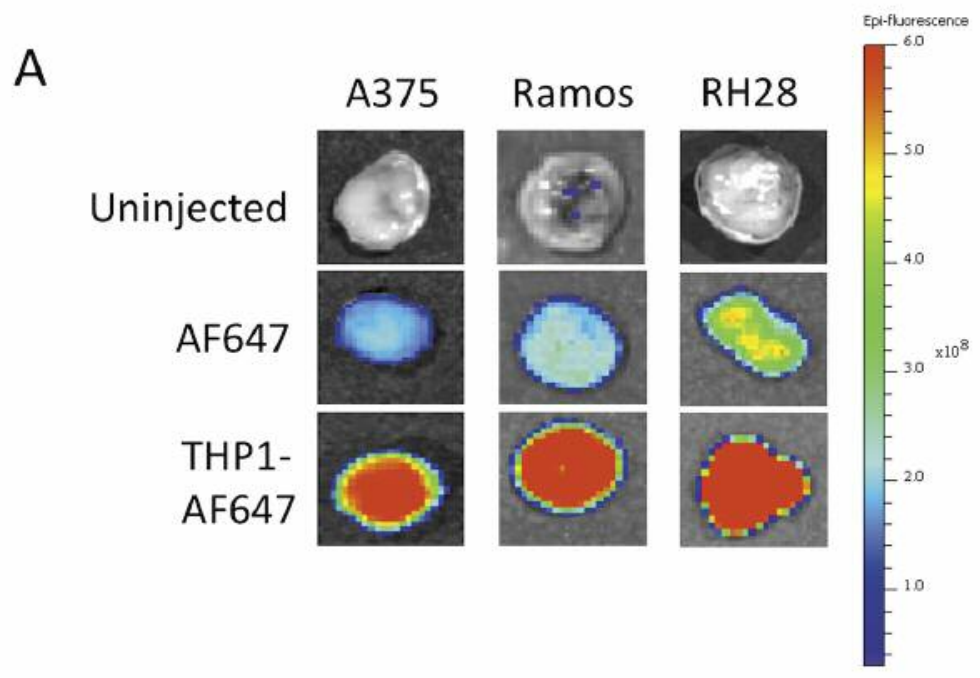

B
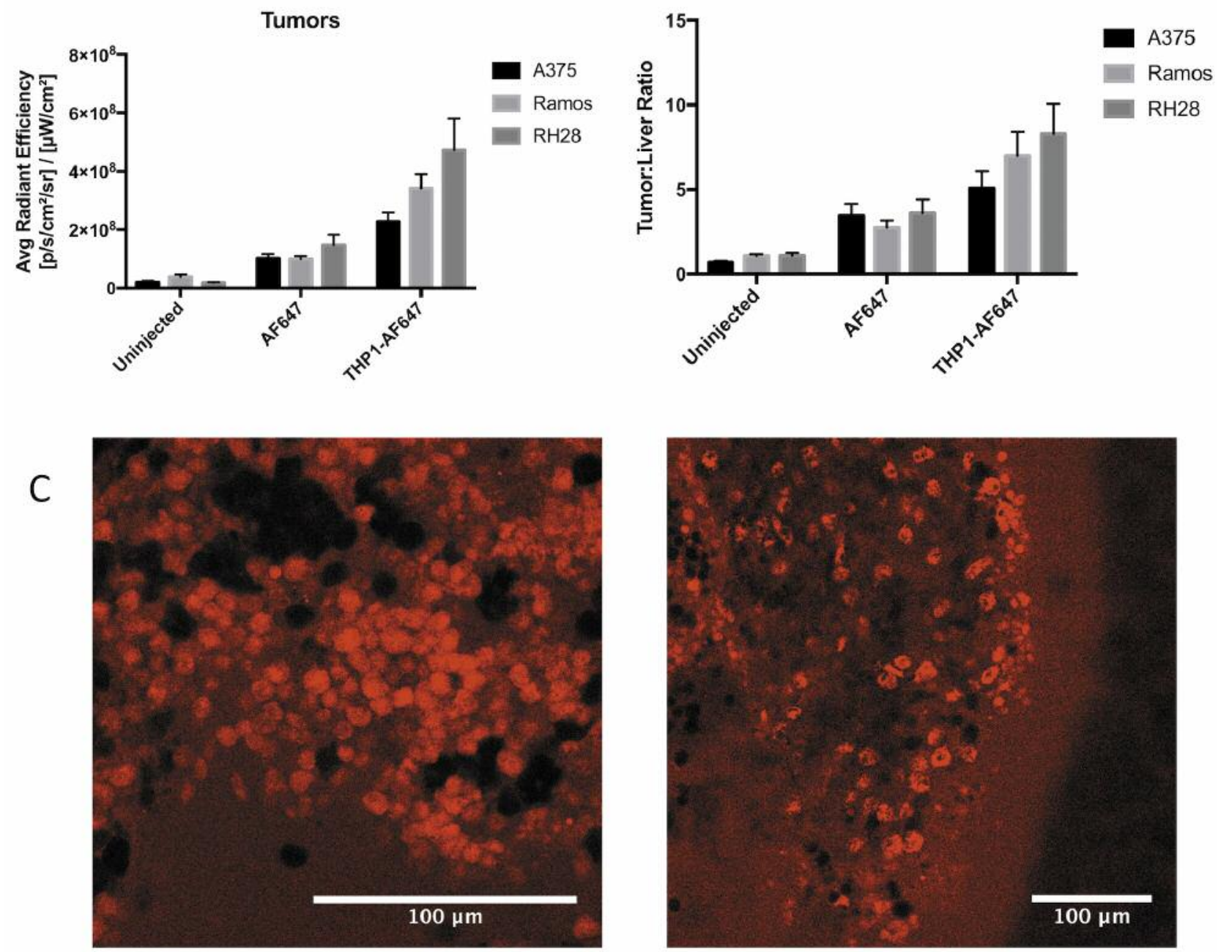

Figure 2. THP1 accumulated in multiple tumor types. A) Images of representative tumors comparing the A375, Ramos, and RH28 tumor lines between the three injection groups. B) Quantitation of signals in tumor and tumor:liver ratios in each of the tumor types. $n=3$. C) Confocal microscopy showing 10 nmol THP1-AF647 internalized in RH28 tumor cells 30 min following in vivo injection. 
A
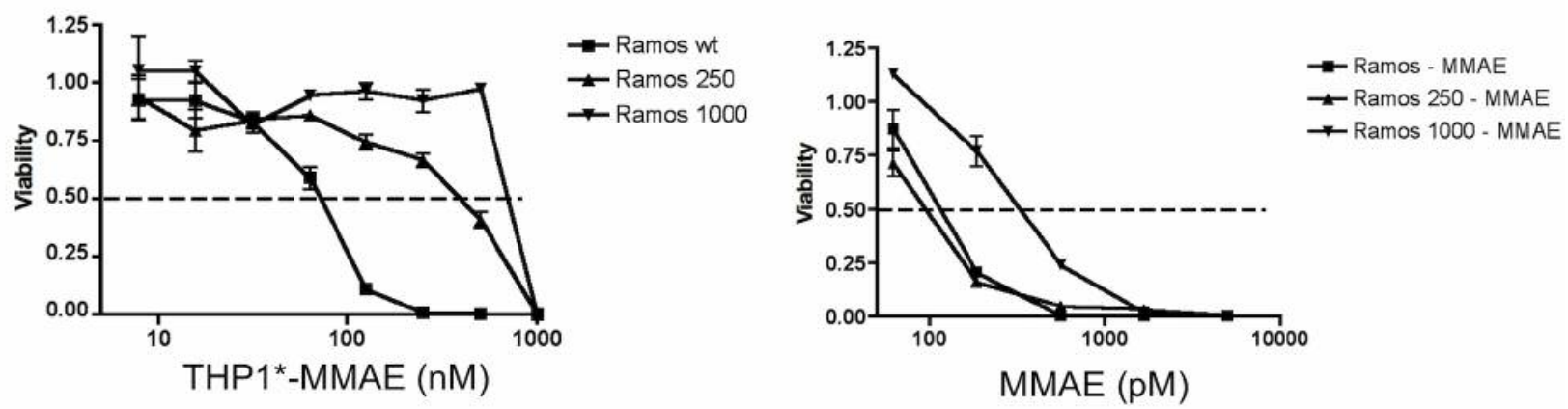

B

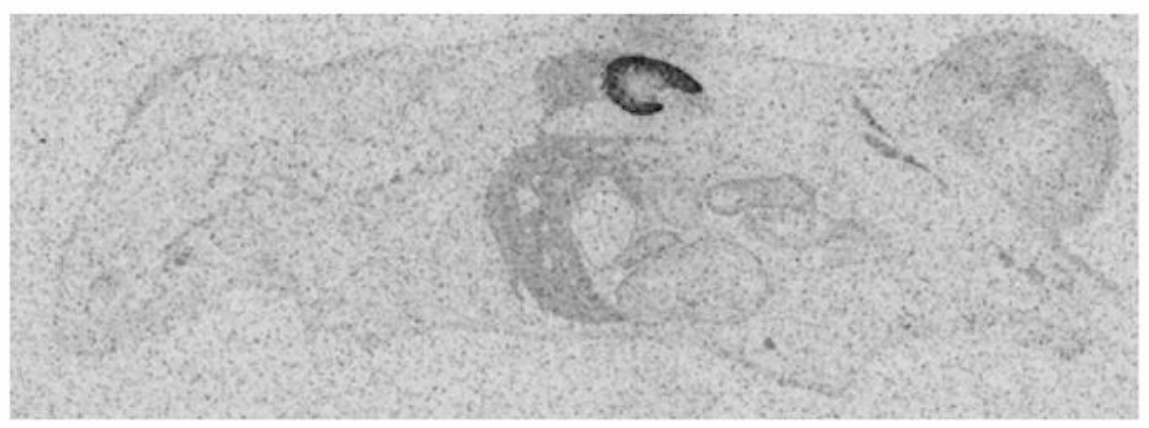

THP1*-MMAE in RH28
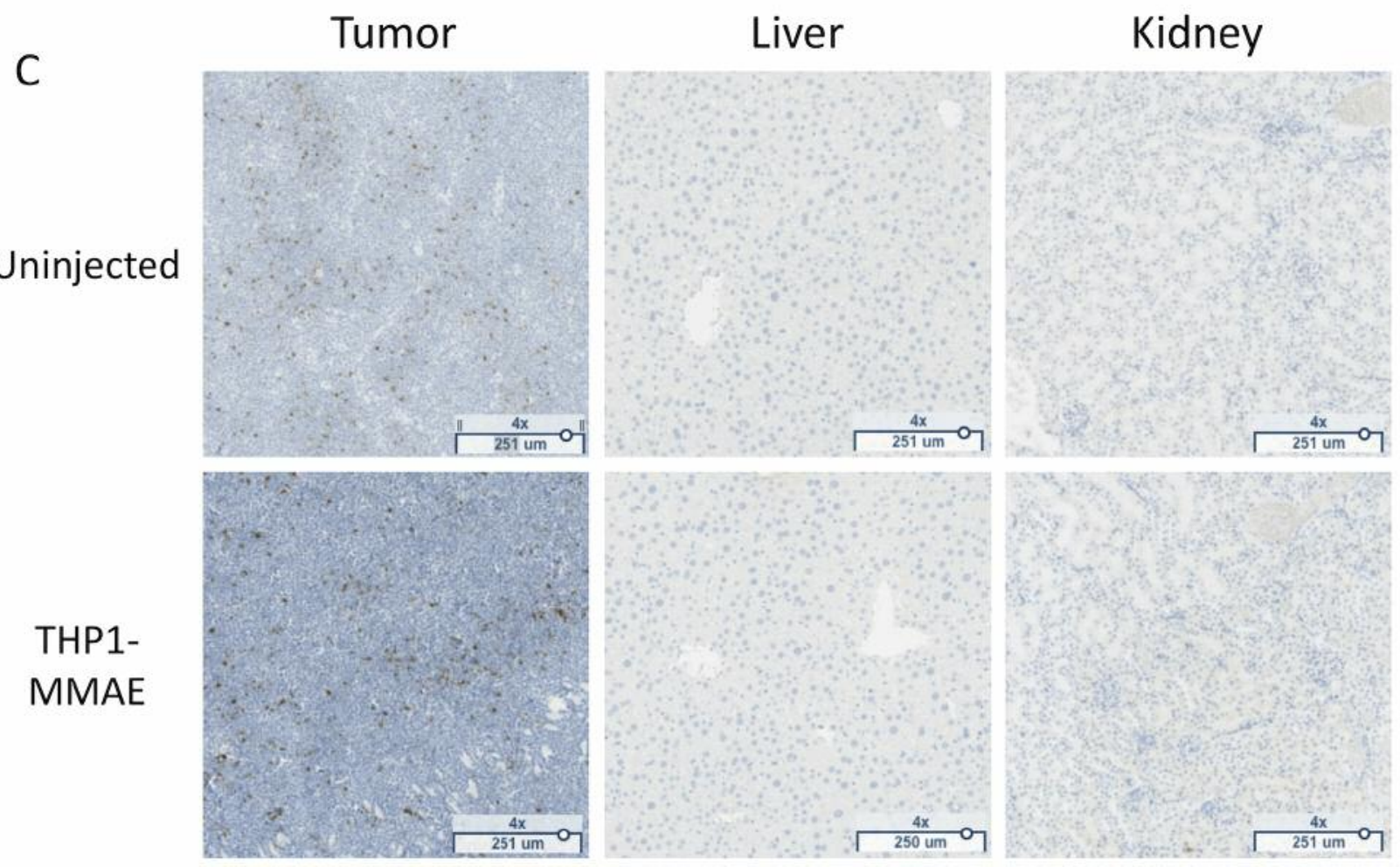

Figure 3. Peptide-drug conjugate was toxic to tumor cells in vitro and in vivo. A) In vitro cell viability assay quantified using CellTiterGlo. Cells were treated for $72 \mathrm{~h}$ and compared to vehicle-treated cells. B) Whole-body autoradiography images demonstrating the biodistribution of THP $1 *$ MMAE at 24 h in mice bearing RH28 flank tumors. C) Immunohistochemistry showing cleaved caspase-3 in select tissues from uninjected mice or mice treated with $100 \mathrm{nmol}$ of THP1*-MMAE conjugate. Mice were treated 48 h prior to necropsy. 
uniform disulfide bonding topology (Figure 1A and B).

To assess the ability of THP1 to deliver an imaging payload to tumors, we labeled THP1 with the dye, Alexa Fluor 647 (AF647). Mice were dosed via tail vein injection with $10 \mathrm{nmol}$ of AF647-labeled THP1 (THP1-AF647) and tissues were imaged ex vivo after $4 \mathrm{~h}$. The dosage and time point were selected based on previous experience demonstrating optimal signal-to-noise in tumor as compared with normal tissue for dyeconjugated chlorotoxin in mouse models. THP1 showed 3.2-fold higher $(p<0.01)$ tumor uptake than AF647 alone in the RH28 sarcoma cell line (Figure 1C). Additionally, tumor specific uptake had an 8.3-fold higher delivery to tumor than to liver. While THP1-AF647 has 60-fold higher fluorescent signal observed in the kidneys when compared with AF647 treated mice this accumulation is often seen following glomerular filtration of small peptides (26). To investigate the biodistribution of the THP1, both with and without conjugation to AF647, we evaluated a further modified form, termed THP $1 *$ and containing a single-lysine (K18), by using whole-body autoradiography. THP1* was prepared with and without a single AF647 conjugate and radiolabeled by reductive-methylation using ${ }^{14} \mathrm{C}$ formaldehyde. Mice bearing RH28 human sarcoma flank tumors were dosed with $2 \mu \mathrm{Ci}$ of a radiolabeled form of THP $1^{*}$ and THP1*-AF647. The mice were subsequently embedded, sectioned, and exposed to phosphoimager plates (Figure 1D). Qualitatively, the biodistribution does not differ when comparing THP1* to THP1*-AF647 in these mice. This demonstrates tumor delivery of both the peptide and peptide-dye conjugate.

We next assessed the ability of THP1-AF647 to accumulate in a panel of tumor types. In RH28 sarcoma, Ramos lymphoma, and A375 melanoma, THP1-AF647 showed a 3.2-, 3.5-, and 2.2-fold higher signal relative to AF647 alone, respectively. Further, the THP1-AF647 signal was 5- to 8-fold higher in tumors compared to liver (Figure $2 \mathrm{~A}$ and $\mathrm{B}$ ). We next determined whether THP1-AF647 is internalized into tumor cells in vivo. Mice were dosed with $10 \mathrm{nmol}$ of THP1-AF647, the tumor was harvested 30 min post-injection to maximize tumor signal, and live tissue was sectioned without fixation and imaged via confocal microscopy. THP1-AF647 indeed penetrated into RH28 cancer cells and was observed as punctate staining inside the majority of tumor cells (Figure 2C). From these data, we conclude that THP1-AF647 accumulates in a number of tumor types and is internalized in tumor cells in vivo.

Peptide internalization into cancer cells is a requirement for any candidate considered for delivery of chemotherapy agents that act on intracellular targets. Given that THP ${ }^{*}$ accumulated inside of cancer cells in vivo, we assessed the ability of this cystine-knotted peptide to deliver chemotherapy as a cleavable peptide-drug conjugate by testing it in a tumor cell line and mouse model. To test whether THP 1 * would be effective as a drug delivery agent, we designed a peptide-drug conjugate using a Val-Cit-PABA (VCP) cleavable linker attached to the microtubule inhibitor monomethyl aurostatin E (MMAE). This

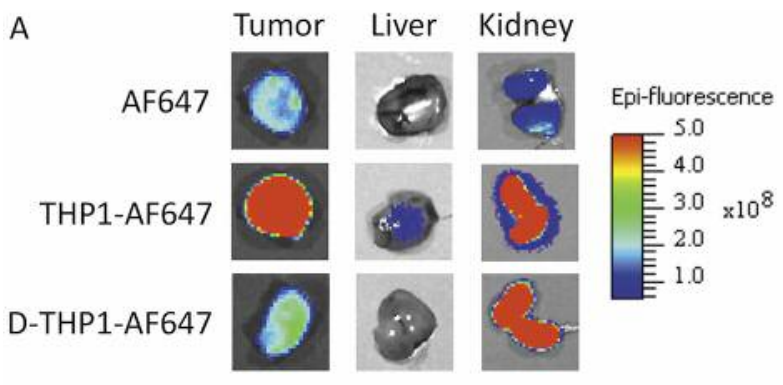

B

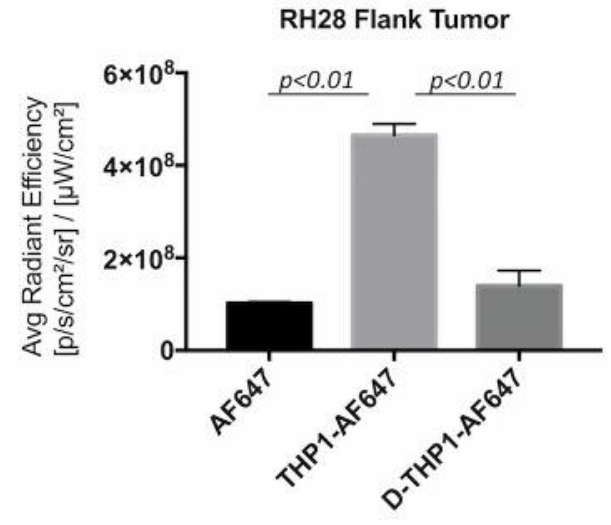

Figure 4. Comparison of the L-vs. D-Amino Acid forms of THP1. A) Mice bearing RH28 flank xenografts were dosed with $10 \mathrm{nmol}$ of AF647, THP1-AF647 or D-THP1-AF647 (D-amino acid peptide) and euthanized 1 h following injection. Select tissues were imaged ex vivo and quantified by IVIS imaging. B) Quantitation of signals in organs, reported in average radiant efficiency. $n=3$.

combination of linker-payload has been shown to have clinical utility in hematopoietic liquid tumors, as the FDA approved brentuximab vedotin (27). MMAE and THP1*-MMAE were tested for their ability to inhibit the growth of Ramos lymphoma cells in vitro. Ramos cells have traditionally been tested with MMAE conjugates because of their sensitivity to microtubule inhibitors (28). MMAE inhibited growth of Ramos cells with $\mathrm{IC}_{50}$ values between 100 and $1000 \mathrm{pM}$, similar to prior reports (Figure 3A). However, THP1*-MMAE was $\sim 1,000$-fold less potent than MMAE alone at growth inhibition of Ramos cells with $\mathrm{IC}_{50}$ values from 100-1,000 $\mathrm{nM}$. This potency reduction is also seen with brentuximab vedotin, which sees potency losses of up to 30-fold in antigen-positive cells when compared with MMAE alone (26). In order to assess activity in models of chemotherapy resistance, we generated Ramos-250 and Ramos1000 sublines (resistance to $250 \mathrm{nM}$ and 1,000 nM doxorubicin, respectively) by continuouly exposing them to increasing concentrations of doxorubicin. Doxorubicin-resistant Ramos sublines (Ramos-250 and Ramos-1000) were resistant to both MMAE and THP1*-MMAE, presumably due to multi-drug resistance mechanisms. While the poor efficacy of THP $1 *_{-}$ MMAE did not bode well for in vivo efficacy, it was still of 
A

LCMI-II
THP1
THP1* (K18)
THP2
THP3
THP4
THP5
THP6
THP7
THP8

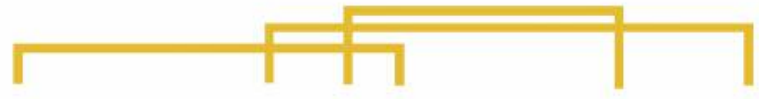

EISCEPGKTFKKDKKCNTCRCGADGKSAACTLKACPNQ GSSCEPGRTFRDRCNTCRCGADGRSAACTLRACPNQ GSSCEPGRTFRDRCNTCKCGADGRSAACTLRACPNQ GSSCEPGTTFRDRCNTCRCGSDGRSAACTLRACPQ GSSCTPGTTFRDRCNTCRCSSNGRSAACTLRACPPGSY GSSCTPGTTFRNRCNTCRCGSNGRSASCTLMACPPGSY GSSCTPGATFRNRCNTCRCGSNGRSASCTLMACPPGSY GSSCQPGTTYQRGCNTCRCLEDGQTEACTLRLC GSSCTPGATYREGCNICRCRSDGRSGACTRRICPVDSN GSSCQPGTTFRRDCNTCVCNRDGTNAACTLRACL

B

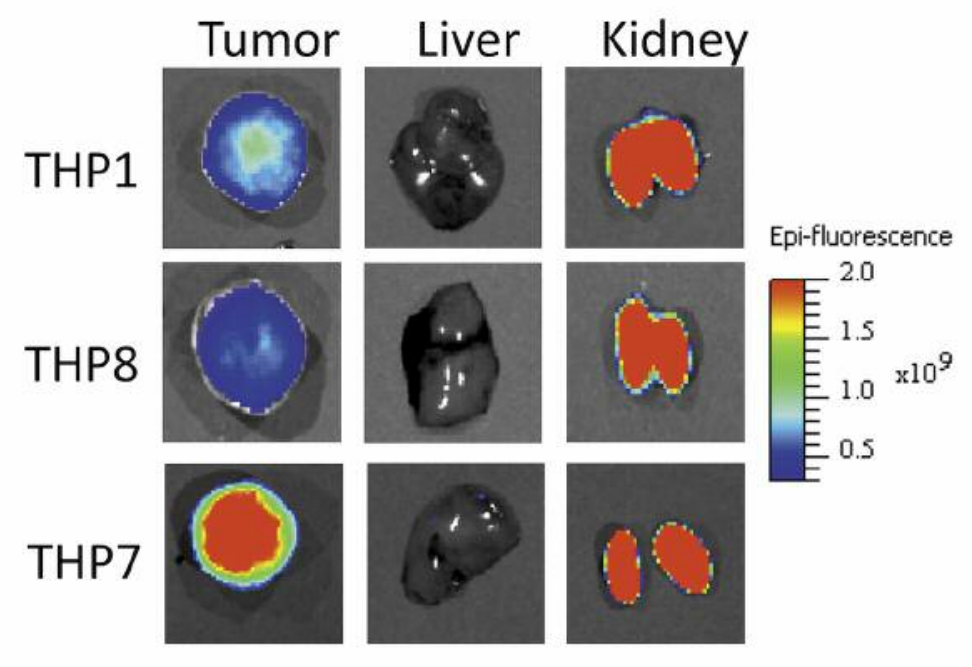

C
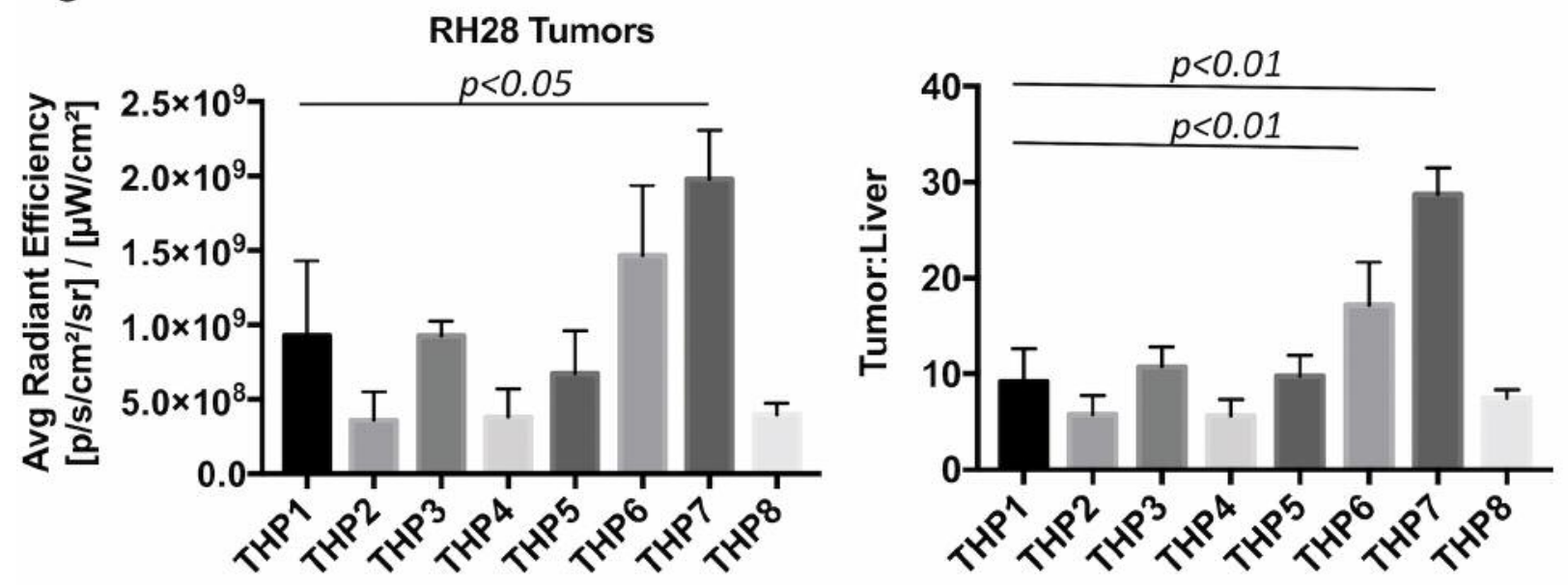

Figure 5. Uptake of THP1 variants in RH28 Flank Tumors. A) Sequences of the native cystine-knotted peptide LCMI-II, modified LCMI-II and variants are shown. Disulfide bonding pattern is illustrated at the top as well as known functional sequence motifs. B) Mice bearing RH28 flank xenografts were dosed with $10 \mathrm{nmol}$ of THP1-AF647 or $10 \mathrm{nmol}$ of THP1 variant-AF647 and euthanized $4 \mathrm{~h}$ following injection. Select tissues were imaged ex vivo and quantified by IVIS imaging. C) Quantitation of signals in tumor and tumor:liver ratios for each of the variant peptides. $n=3-6$. 
interest to learn whether THP1* could deliver a candidate chemotherapeutic agent to tumors in vivo or whether targeting activity was impaired by the payload.

To evaluate the biodistribution of THP $1 *$-MMAE, the molecule was ${ }^{14} \mathrm{C}$ methylated, and $2 \mu \mathrm{Ci}$ were administered to mice bearing Ramos flank tumors. After $24 \mathrm{~h}$, the mice were euthanized, sectioned, and exposed to phosphoimager plates (Figure 3B). The MMAE bioconjugate accumulation in tumors and normal tissues was similar to radiolabeled THP1 alone; however, the overall signal of the bioconjugate was 3- to 4-fold lower than radiolabeled THP1 in all tissues. Due to the low levels of bioconjugate accumulation in the tumors in vivo, we turned to immunohistochemistry as a more sensitive measure of pathway engagement. Following exposure to THP1*-MMAE, we examined whether the apoptotic pathways (as measured by cleaved caspase 3 signal) were activated in Ramos tumor cells in vivo. Mice were treated for $48 \mathrm{~h}$ with $100 \mathrm{nmol}$ of THP1*MMAE before tissues were harvested and assessed for cleaved caspase-3 (CC3) by immunohistochemistry (Figure 3C). Not surprisingly, scattered $(<10 \%) \mathrm{CC} 3$ was seen in the tumor of both control and drug-treated mice with little to no $(<5 \%) \mathrm{CC} 3$ seen in liver or kidney. Together, these data indicate that while THP1 is delivered to tumors, THP1-MMAE is minimally active in vitro and in vivo.

To address whether the mechanism of THP1 accumulation in tumors is driven by peptide charge and size, an all D-amino acid form of THP1 was synthesized and termed D-THP1. We then tested for accumulation in RH28 flank tumors. Mice were dosed IV with 10 nmol AF647, THP1-AF647, or D-THP1AF647, and tissues were imaged ex vivo after $1 \mathrm{~h}$, as earlier time points demonstrated a greater ability to detect signal decreases in the tumor (Figure 4). THP1-AF647 had a significant $(p<0.01)$ increased tumor accumulation when compared with D-THP1-AF647. The enhanced tumor accumulation found in THP1-AF647 (in comparison to the Damino acid form) suggests that enhanced accumulation resulted from the peptide sequence rather than size or charge, and led us to explore sequence variants of THP1.

A panel of 27 variants were generated to characterize the ability of THP1 to act as a scaffold for peptide engineering. Mutations focused on: 1) charge-reversal point mutations; 2) mutation of an asparagine residue critical for folding; and 3) using peptide sequences from close homologs identified by BLAST. This panel was assessed for by HPLC under nonreduced and reduced conditions and characterized as either a "well-folded" or "mixed" product. Sixteen of the 27 tested peptides were well-folded and formed single products by HPLC, which shifted upon reduction; 7 of the 16 were based on homologous peptides and 9 were point mutations. We tested the 7 well-folded homologous peptides, with 50-90\% amino acid sequence homology when compared to THP, for their ability to enhance tumor accumulation in RH28 human sarcoma flank tumors (Figure 5). Mice were dosed IV with $10 \mathrm{nmol}$ of peptide-

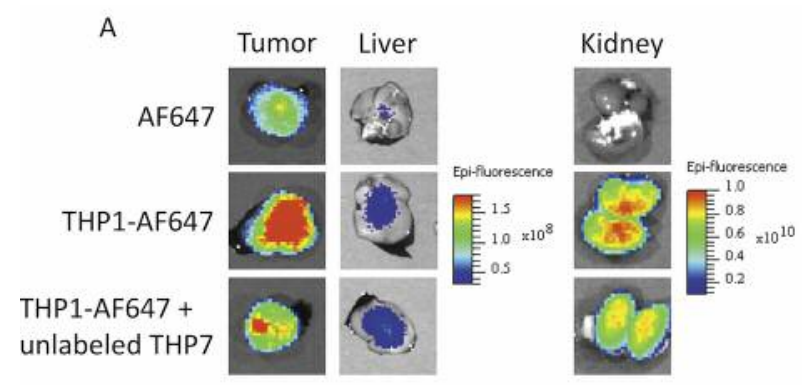

B

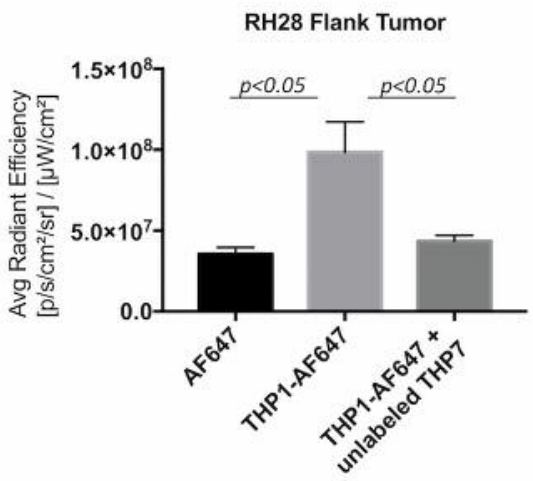

Figure 6. In vivo competition between THP1 and THP7. A) Mice bearing RH28 flank xenografts were dosed with $2 \mathrm{nmol}$ of AF647, 2 nmol of THP1-AF647, or 2 nmol of THP1-AF647 with 98 nmol of unlabeled THP7 and euthanized 1 hour following injection. Select tissues were imaged ex vivo. B) Quantitation of signals in organs, reported in average radiant efficiency. $n=3$.

AF647 conjugate and tissues were imaged ex vivo after $4 \mathrm{~h}$. Tumor accumulation of THP7-AF647 increased 2.1-fold $(p<0.05)$ in comparison to THP1-AF647. Additionally, tumorto-liver ratios were elevated 1.9 -fold $(p<0.05)$ and 3.1 -fold $(p<0.01)$ compared to THP1-AF647 for THP6-AF647 and THP7-AF647, respectively. To test whether these peptides hit the same molecular target in a competitive manner, mice were dosed with AF647, THP1-AF647, or both THP1-AF647 and a 50-fold excess of unlabeled THP7 (Figure 6). Tissues were imaged $e x$ vivo and tumor signal was decreased 2.2-fold $(p<0.05)$ following the addition of the unlabeled competitor, THP7. This suggests that the yet to be identified molecular target is shared and binds to these peptides in a competitive manner.

\section{Discussion}

In this study, we identified a novel peptide-dye conjugate derived from the LCMI-II protease inhibitor that accumulates in multiple cancer types. Whole-body autoradiography showing qualitatively similar distribution of THP1* with or without a dye conjugate suggests that the peptide plays a dominant role in biodistribution, and that a payload, such as AF647, does not interfere with tumor localization. We determined that the 
accumulation of the THP1-AF647 bioconjugate is both stereospecific and saturable. We identified modified forms that accumulate in tumors with more favorable ratios of tumor-tonormal tissues (e.g. liver tissue).

LCMI-II is highly tolerant to mutation, as has been seen in cystine-knotted peptides such as EETI, MCoTI, AgRP, and others (29-31), with some residues more tolerant of mutation than others. We have identified the sensitivity to mutagenesis of a number of residues in the THP1 scaffold. These variants of THP1 were assessed for tumor accumulation, and THP7 was identified as a variant with significantly enhanced tumor accumulation. Additionally, THP7 showed a tumor:liver ratio of 30:1, demonstrating high specificity. Tumor accumulation of the variants and the observed competition between THP1 and THP7 has shown us certain residues of LCMI-II-related peptides that are important for tumor accumulation.

The binding of THP1 on the tumor occurs via a competitive and stereospecific process, suggesting that THP1 is targeting a specific protein receptor on these cells. The native sequence of LCMI-II is a high affinity inhibitor of both chymotrypsin and elastase. Of the candidate protein receptors, the most intriguing is the potential for pacifastins to bind to cancer-associated serine proteases. The structure of chymotrypsin in its co-crystal structure with LCMI-II has a highly-conserved backbone and binding site in comparison to cancer-associated serine proteases (32). Specifically, the type II transmembrane serine proteases matriptase, hepsin, TMPRSS2, and TMPRSS4 have been found to be overexpressed in many cancers, predominantly studied in prostate, breast, and ovarian cancers (33). Binding to these transmembrane proteins has the potential to trigger endocytosis and internalization of peptide-dye and peptide-drug conjugates that lead to payload delivery. While beyond the scope of the current work described here, in the event that THP1 is developed for imaging or therapeutic purposes, it will be necessary to distinguish between these possibilities.

We conducted proof-of-concept experiments with THP1MMAE bioconjugates to assess whether the peptide-drug conjugate is able to release a drug and trigger cell death. Despite observing cell death in vitro, the fraction of apoptotic cells in THP1-MMAE-treated tumors in vivo was no different than vehicle controls. The lack of efficacy observed with the delivery of MMAE or other potent drugs may be a result of: 1) short serum half-life of the peptide-drug conjugate; 2) delivery of an insufficient number of MMAE molecules to be therapeutically effective (THP $1 *$ carries only one payload, whereas antibodydrug conjugates typically carry 4-8 payloads); 3 ) premature release of the drug in serum during distribution; 4) reduced potency of the bioconjugate (e.g., incomplete linker cleavage); or 5) intracellular trafficking routes, such as endosomal trapping, that prevent MMAE from reaching microtubules. The reduced potency of THP1-MMAE compared to unconjugated MMAE in vitro suggests that drug release is also an issue. Future efforts toward therapeutic peptide conjugates will require significant work on pharmacokinetic optimization (e.g., extending serum half-life) and potency (e.g., providing a carrier of multiple MMAE or therapeutic payloads). Additional work on intracellular trafficking will also be necessary to understand whether the payload has access to its target.

In our study, our goal was to identify a new cystine-knotted peptide that accumulates in tumors. In future experiments, we plan on identifying ways of extending the half-life of peptidedrug conjugates to increase their exposure to tumors. Additionally, we plan to utilize computational design to generate high-affinity ligands for the cancer-associated proteases based on our starting scaffold THP1. In our work described here, we have shown the potential for using LCMIII-derived cystine-knotted peptide to deliver imaging agents to tumors, and we have established the foundation for the development of peptide-drug conjugates.

\section{Conflicts of Interest}

J.M.O. is a founder and shareholder of Blaze Bioscience, Inc.

\section{Acknowledgements}

This study was supported by NIH/NCI grant R01CA135491 and Project Violet. T.S. was supported by the Anthony and Julianne Capetola Fellowship and the ARCS Foundation. The Authors would like to thank Kyle Pedro, Midori Clarke, Albe Chan, Willem de van der Schueren, Ryan Steele, Mesfin Gewe, Kannan Karukurichi, Jane Carter, Fiona Pakiam, and Ashok Bandaranayake for experimental support and Roland Strong, Natalie Nairn, Zachary Crook, Chris Mehlin, Colin Correnti, and Andy Strand for helpful discussion. The data that support the findings of this study are available from the authors on reasonable request.

\section{References}

1 Goodman LS, Wintrobe MM and Dameshek W: Nitrogen mustard therapy; use of methyl-bis (beta-chloroethyl) amine hydrochloride and tris (beta-chloroethyl) amine hydrochloride for hodgkin's disease, lymphosarcoma, leukemia and certain allied and miscellaneous disorders. J Am Med Assoc 132: 126-132, 1946.

2 Lacroix M, Abi-Said D, Fourney DR, Gokaslan ZL, Shi W, DeMonte F, Lang FF, McCutcheon IE, Hassenbusch SJ, Holland E, Hess K, Michael C, Miller D and Sawaya R: A multivariate analysis of 416 patients with glioblastoma multiforme: Prognosis, extent of resection, and survival. J Neurosurg 95(2): 190-198, 2001.

3 Daigeler A, Zmarsly I, Hirsch T, Goertz O, Steinau HU, Lehnhardt $\mathrm{M}$ and Harati K: Long-term outcome after local recurrence of soft tissue sarcoma: A retrospective analysis of factors predictive of survival in 135 patients with locally recurrent soft tissue sarcoma. Br J Cancer 110(6): 1456-1464, 2014.

4 van Tinteren H, Hoekstra OS, Smit EF, van den Bergh JH, Schreurs AJ, Stallaert RA, van Velthoven PC, Comans EF, Diepenhorst FW, Verboom P, van Mourik JC, Postmus PE, Boers $M$ and Teule GJ: Effectiveness of positron emission tomography in the preoperative assessment of patients with suspected nonsmall-cell lung cancer: The plus multicentre randomised trial. Lancet 359(9315): 1388-1393, 2002. 
5 Caravan P, Ellison JJ, McMurry TJ and Lauffer RB: Gadolinium(iii) chelates as mri contrast agents: Structure, dynamics, and applications. Chem Rev 99(9): 2293-2352, 1999.

6 Young H, Baum R, Cremerius U, Herholz K, Hoekstra O, Lammertsma AA, Pruim J and Price P: Measurement of clinical and subclinical tumour response using $\left[{ }^{18} \mathrm{~F}\right]$-fluorodeoxyglucose and positron emission tomography: Review and 1999 eortc recommendations. European organization for research and treatment of cancer (eortc) pet study group. Eur J Cancer 35(13): 1773-1782, 1999.

7 Thurber GM, Schmidt MM and Wittrup KD: Factors determining antibody distribution in tumors. Trends Pharmacol Sci 29(2): 57-61, 2008.

8 Adams DJ: The valley of death in anticancer drug development: A reassessment. Trends Pharmacol Sci 33(4): 173-180, 2012.

9 Wittrup KD, Thurber GM, Schmidt MM and Rhoden JJ: Practical theoretic guidance for the design of tumor-targeting agents. Methods Enzymol 503: 255-268, 2012.

10 Kapoor P, Singh H, Gautam A, Chaudhary K, Kumar R and Raghava GP: Tumorhope: A database of tumor homing peptides. PLoS One 7(4): e35187, 2012.

11 Parrish-Novak J, Byrnes-Blake K, Lalayeva N, Burleson S, Fidel J, Gilmore R, Gayheart-Walsten P, Bricker GA, Crumb WJ, Jr., Tarlo KS, Hansen S, Wiss V, Malta E, Dernell WS, Olson JM and Miller DM: Nonclinical profile of blz-100, a tumor-targeting fluorescent imaging agent. Int J Toxicol 36(2): 104-112, 2017.

12 Kuai R, Yuan W, Li W, Qin Y, Tang J, Yuan M, Fu L, Ran R, Zhang $\mathrm{Z}$ and He Q: Targeted delivery of cargoes into a murine solid tumor by a cell-penetrating peptide and cleavable poly(ethylene glycol) comodified liposomal delivery system via systemic administration. Mol Pharm 8(6): 2151-2161, 2011

13 Kolmar H: Biological diversity and therapeutic potential of natural and engineered cystine knot miniproteins. Curr Opin Pharmacol 9(5): 608-614, 2009.

14 Moore SJ, Hayden Gephart MG, Bergen JM, Su YS, Rayburn H, Scott MP and Cochran JR: Engineered knottin peptide enables noninvasive optical imaging of intracranial medulloblastoma. Proc Natl Acad Sci USA 110(36): 14598-14603, 2013.

15 Veiseh M, Gabikian P, Bahrami SB, Veiseh O, Zhang M, Hackman RC, Ravanpay AC, Stroud MR, Kusuma Y, Hansen SJ, Kwok D, Munoz NM, Sze RW, Grady WM, Greenberg NM, Ellenbogen RG and Olson JM: Tumor paint: A chlorotoxin:Cy5.5 bioconjugate for intraoperative visualization of cancer foci. Cancer Res 67(14): 6882-6888, 2007

16 Craik DJ, Daly NL and Waine C: The cystine knot motif in toxins and implications for drug design. Toxicon 39(1): 43-60, 2001.

17 Gerlinger M, Rowan AJ, Horswell S, Math M, Larkin J, Endesfelder D, Gronroos E, Martinez P, Matthews N, Stewart A, Tarpey P, Varela I, Phillimore B, Begum S, McDonald NQ, Butler A, Jones D, Raine K, Latimer C, Santos CR, Nohadani M, Eklund AC, Spencer-Dene B, Clark G, Pickering L, Stamp G, Gore M, Szallasi Z, Downward J, Futreal PA and Swanton C: Intratumor heterogeneity and branched evolution revealed by multiregion sequencing. N Engl J Med 366(10): 883-892, 2012.

18 Bedard PL, Hansen AR, Ratain MJ and Siu LL: Tumour heterogeneity in the clinic. Nature 501(7467): 355-364, 2013.

19 Breugelmans B, Simonet G, van Hoef V, Van Soest S and Vanden Broeck J: Pacifastin-related peptides: Structural and functional characteristics of a family of serine peptidase inhibitors. Peptides 30(3): 622-632, 2009.
20 Simonet G, Claeys I and Broeck JV: Structural and functional properties of a novel serine protease inhibiting peptide family in arthropods. Comp Biochem Physiol B Biochem Mol Biol 132(1): 247-255, 2002.

21 Kellenberger C, Boudier C, Bermudez I, Bieth JG, Luu B and Hietter $\mathrm{H}$ : Serine protease inhibition by insect peptides containing a cysteine knot and a triple-stranded beta-sheet. J Biol Chem 270(43): 25514-25519, 1995.

22 Boigegrain RA, Mattras H, Brehelin M, Paroutaud P and ColettiPreviero MA: Insect immunity: Two proteinase inhibitors from hemolymph of locusta migratoria. Biochem Biophys Res Commun 189(2): 790-793, 1992.

23 Nakakura N, Hietter H, Van Dorsselaer A and Luu B: Isolation and structural determination of three peptides from the insect locusta migratoria. Identification of a deoxyhexose-linked peptide. Eur J Biochem 204(1): 147-153, 1992.

24 Bandaranayake AD, Correnti C, Ryu BY, Brault M, Strong RK and Rawlings DJ: Daedalus: A robust, turnkey platform for rapid production of decigram quantities of active recombinant proteins in human cell lines using novel lentiviral vectors. Nucleic Acids Res 39(21): e143, 2011.

25 Jentoft $\mathrm{N}$ and Dearborn DG: Labeling of proteins by reductive methylation using sodium cyanoborohydride. J Biol Chem 254(11): 4359-4365, 1979.

26 Carone FA: Renal handling of proteins and peptides. Ann Clin Lab Sci 8(4): 287-294, 1978.

27 Younes A, Bartlett NL, Leonard JP, Kennedy DA, Lynch CM, Sievers EL and Forero-Torres A: Brentuximab vedotin (sgn-35) for relapsed cd30-positive lymphomas. N Engl J Med 363(19): 1812-1821, 2010.

28 Francisco JA, Cerveny CG, Meyer DL, Mixan BJ, Klussman K, Chace DF, Rejniak SX, Gordon KA, DeBlanc R, Toki BE, Law CL, Doronina SO, Siegall CB, Senter PD and Wahl AF: Cac10-vcmmae, an anti-cd30-monomethyl auristatin e conjugate with potent and selective antitumor activity. Blood 102(4): 1458-1465, 2003.

29 Kimura RH, Levin AM, Cochran FV and Cochran JR: Engineered cystine knot peptides that bind alphavbeta3, alphavbeta5, and alpha5beta1 integrins with low-nanomolar affinity. Proteins 77(2): 359-369, 2009.

30 Quimbar P, Malik U, Sommerhoff CP, Kaas Q, Chan LY, Huang YH, Grundhuber M, Dunse K, Craik DJ, Anderson MA and Daly NL: High-affinity cyclic peptide matriptase inhibitors. J Biol Chem 288(19): 13885-13896, 2013.

31 Silverman AP, Levin AM, Lahti JL and Cochran JR: Engineered cystine-knot peptides that bind alpha(v)beta(3) integrin with antibody-like affinities. J Mol Biol 385(4): 1064-1075, 2009.

32 Roussel A, Mathieu M, Dobbs A, Luu B, Cambillau C and Kellenberger C: Complexation of two proteic insect inhibitors to the active site of chymotrypsin suggests decoupled roles for binding and selectivity. J Biol Chem 276(42): 38893-38898, 2001.

33 Murray AS, Varela FA and List K: Type ii transmembrane serine proteases as potential targets for cancer therapy. Biol Chem 397(9): 815-826, 2016. 